\title{
Thermal entanglement of spins in mean-field clusters
}

\author{
M. Asoudeh ${ }^{1}$ V. Karimipour ${ }^{2}$ \\ Department of Physics, Sharif University of Technology, \\ P.O. Box 11365-9161, \\ Tehran, Iran
}

\begin{abstract}
We determine thermal entanglement in mean field clusters of $N$ spin one-half particles interacting via the anisotropic Heisenberg interaction, with and without external magnetic field. For the $x x x$ cluster in the absence of magnetic field we prove that only the $N=2$ ferromagnetic cluster shows entanglement. An external magnetic field $B$ can only entangle $x x x$ anti-ferromagnetic clusters in certain regions of the $B-T$ plane. On the other hand, the $x x z$ clusters of size $N>2$ are entangled only when the interaction is ferromagnetic. Detailed dependence of the entanglement on various parameters is investigated in each case.
\end{abstract}

PACS Numbers: 03.65.Ud, 05.50.+9, 75.10.Jm.

\footnotetext{
${ }^{1}$ email:asoudeh@mehr.sharif.edu

${ }^{2}$ Corresponding author, email:vahid@sharif.edu
} 


\section{Introduction}

It is usually stated that at any finite non-zero temperature, thermal fluctuations suppress quantum fluctuations and for that reason the latter can exist only at absolute zero or very low temperatures. With the progress in quantifying entanglement [1] or quantum correlations, we have now a precise method for determining exactly at what temperatures quantum correlations cease to exist. This problem has been investigated extensively under the name "Thermal Entanglement" in many body quantum systems [2, 3, 4, 15, 6, 17, 8, 9, 10, 11, 12, 13, 14, 15, 16]. The term is usually used to specify the amount of entanglement or genuine quantum correlation which exists between two parts of a system when the whole system is in a state of thermal equilibrium. One is interested in how this quantity depends on the coupling constants of the system and the temperature. Usually there is a threshold temperature above which entanglement vanishes. In some rare cases it is even observed that raising the temperature first increases the quantum correlation and then begins to diminish it [3]. In this way by a quantitative treatment of the problem we are able to sharpen our intuitive notions on the effect of temperature on quantum correlations.

In addition to these purely theoretical considerations, these works are also motivated by the proposals in which spins in solid state systems play the role of qubits [17, 18, 19, 20, 21, 22, 23. and the fact that thermal entanglement seems to be stable against de-coherence and needs no controlled switching for its generation.

In this regard in the past few years many types of spin systems have been studied, which include, the Heisenberg rings with ferromagnetic and anti-ferromagnetic coupling in the absence and presence of magnetic fields, small clusters of spins with different types of isotropic and anisotropic coupling in homogeneous and in-homogeneous magnetic fields. These are only a small sample of the works which have been done in this direction. There are also other works which take into account other forms of interactions between the spins.

Obtaining exact results on thermal entanglement in spin systems on arbitrary lattices is extremely difficult, since this generally requires a knowledge of different correlation functions which in turn requires a knowledge of the full spectrum of the system. However in certain systems with extra symmetries one can obtain some partial and interesting results [6].

In this paper we want to study in some considerable detail thermal entanglement of spins in mean field clusters of arbitrary sizes. These are clusters in which every spin is coupled to every other spin with equal strength. Such an study has a two-fold motivation. First, mean field systems are amenable to exact analytical treatment since as we will see, one can determine the entanglement by the sole knowledge of energy eigenvalues, i.e. the partition function, and second they are the first approximation that one uses to study other models. Therefore the results which are obtained for entanglement in these systems, may be good approximations for their corresponding results on other lattices specially when the coordination number of the 
latter is high.

We would like to stress that in all these considerations we are concerned with the thermal state of the system which at zero temperature approaches an equal mixture of various ground states, if there is a degeneracy in the spectrum. For the entanglement properties of the ground states of $x y$ mean field clusters the reader is referred to [24, 25, 27, 28, 29, 30].

In these works a general class of mean field models under the name collective models have been considered and the entanglement properties of their ground states with particular emphasis to its relation with quantum phase transitions have been studied.

The structure and the results of this paper are as follows: In section 2 we introduce the model which consists of a system of $N$ spin one-half particles interacting with each other and placed in an external magnetic field. The interaction may be of ferromagnetic or antiferromagnetic type and there may also be present some degree of anisotropy in the couplings of spins in different directions. We obtain general formulas for the thermal entanglement of this model derived from its partition function. In the following sections we study in detail special cases of the model, that is, in section 3 we consider the isotropic (or $x x x$ model) without magnetic field for both ferromagnetic and anti-ferromagnetic interactions, where we show that only the anti-ferromagnetic cluster of $N=2$ spins shows entanglement. In section 4 we study the effect of magnetic field in the $x x x$ model and show that the ferromagnetic clusters show no entanglement while anti-ferromagnetic clusters have entanglement in certain regions of the $B-T$ plane, the plane of magnetic field and temperature. Finally in section 5 we consider the anisotropic ( $x x z$ model) without magnetic field. For an $N=2$ cluster both the ferromagnetic and anti-ferromagnetic interactions produce entanglement, however remarkably for $N>2$ we see that only ferromagnetic clusters show entanglement and no entanglement develops in antiferromagnetic clusters. We determine the regions in the $\Delta-T$ plane, the plane of the anisotropy and temperature where entanglement is non zero. In all cases we determine the dependence of the entanglement in terms of the size of the cluster. Needless to say in a mean field cluster the entanglement between any two spins is very low compared to the chain, due to the large number of neighbors of a site. Therefore we use the re-scaled concurrence [25], the ordinary concurrence scaled by the number of neighbors to measure the degree of entanglement of a cluster.

\section{Interacting spins on mean field graphs}

Consider a cluster of spin $\frac{1}{2}$ particles in an external magnetic field and interacting with each other. We take the Hamiltonian as

$$
H=\frac{J}{N-1} \sum_{i \neq j=1}^{N}\left(\vec{s}_{i} \cdot \vec{s}_{j}+\Delta s_{i z} s_{j z}\right)+B \sum_{i=1}^{N} s_{i z}
$$


where $s_{i a}=\frac{1}{2} \sigma_{i a}$ and $\sigma_{i a}$ 's are Pauli operators. The coupling constant is taken as $\frac{J}{N-1}$ to ensure an extensive total energy. We also set $J=-1$ for ferromagnetic and $J=+1$ for antiferromagnetic clusters and without loss of generality $B$ is taken positive. The parameter $\Delta$ determines the anisotropy of the interaction. For $\Delta=0$ we have an isotropic ( $x x x$ system which in the absence of external magnetic field displays full $S U(2)$ symmetry. This system is exactly solvable since one can rewrite it in terms of the total spin operators $S_{a}:=\sum_{i} s_{i a}$ in the form

$$
H=\frac{J}{N-1}\left(\vec{S} \cdot \vec{S}+\Delta S_{z}^{2}\right)+B S_{z},
$$

where $S^{2}=S_{x}^{2}+S_{y}^{2}+S_{z}^{2}$ and we have ignored an additive constant. Note that in studying the ground state properties where one can restrict to a fixed spin sector (i.e. $S_{x}^{2}+S_{y}^{2}+S_{z}^{2}=S^{2}$ ), the model is equivalent to the Lipkin-Meshkov-Glick model [26] which has been extensively studied in [27, 28, 29]. However for the thermal entanglement properties of the model one should take into account all spin sectors and the above equivalence no longer holds.

For any system of spins having the symmetry $\left[S_{z}, H\right]=0$, it has been shown [1, 6] that the reduced density matrix between two spins is given by

$$
\rho_{i j}=\left(\begin{array}{cccc}
u^{+} & & & \\
& w_{1} & z^{*} & \\
& z & w_{2} & \\
& & & u^{-}
\end{array}\right),
$$

where

$$
\begin{aligned}
u^{ \pm} & =\frac{1}{4} \pm \mu+G_{z z} \\
z & =G_{x x}+G_{y y}+i\left(G_{x y}-G_{y x}\right)
\end{aligned}
$$

and we have ignored the labels indicating the two sites in question which for the mean field cluster can be any two sites. Here $G_{\alpha \beta}$ and $\mu$ are the spin-spin correlation functions and the magnetization per site respectively.

$$
\begin{aligned}
G_{\alpha \beta} & =\left\langle s_{i \alpha} s_{j \beta}\right\rangle, \\
\mu & =\left\langle s_{i z}\right\rangle=\frac{1}{N}\left\langle S_{z}\right\rangle .
\end{aligned}
$$

(Note that there is slight difference between our notations and that of [6] who take $G_{a b}=$ $\left.\left\langle\sigma_{a} \sigma_{b}\right\rangle\right)$. For such a density matrix the concurrence is given by [1, 6]

$$
C=2 \max \left[0,|z|-\sqrt{u^{+} u^{-}}\right] .
$$

from which one can obtain the entanglement of formation (Eof) from

$$
\operatorname{Eof}(\rho)=-\lambda \log \lambda-(1-\lambda) \log (1-\lambda),
$$


with $\lambda=\frac{1-\sqrt{1-C^{2}}}{2}$. We now note that $z$ is real. This can be proved by resorting to the reality of the Hamiltonian and hence the reality of the total and the reduced density matrices. It is worth mentioning that when the interaction strengths between the $x$, and the $y$ terms are not equal, the symmetry $\left[H, S_{z}\right]=0$ will no longer hold and the reduced density matrix will not be of the form (3). For these cases one can resort to a recent conjecture of [27] who provide the closed form of the concurrence for general models having permutation symmetry.

Thus the concurrence can be expressed solely in terms of the correlation functions

$$
C=2 \max \left[0,\left|G_{x x}+G_{y y}\right|-\sqrt{\left(\frac{1}{4}+G_{z z}\right)^{2}-\mu^{2}}\right] .
$$

The magnetization per site $\mu$, and the energy per site $\varepsilon:=\frac{\langle H\rangle}{N}$ are obtained from the partition function as follows:

$$
\begin{aligned}
\mu & =-\frac{1}{N \beta} \frac{\partial \ln Z}{\partial B} \\
\varepsilon & =-\frac{1}{N} \frac{\partial \ln Z}{\partial \beta}
\end{aligned}
$$

The correlation function $G_{z z}$ is obtained from either of the following relations:

$$
\begin{aligned}
G_{z z} & =\frac{1}{N(N-1)}\left[\frac{1}{\beta^{2} Z} \frac{\partial^{2} Z}{\partial B^{2}}-\frac{N}{4}\right], \\
G_{z z} & =-\frac{1}{N J \beta} \frac{\partial}{\partial \Delta} \ln Z-\frac{1}{4(N-1)} .
\end{aligned}
$$

The other correlation function $G_{x x}+G_{y y}$ is obtained from

$$
\varepsilon=\frac{\langle H\rangle}{N}=J\left(G_{x x}+G_{y y}+(1+\Delta) G_{z z}\right)+\mu B
$$

where we have used the exchange symmetry between all the pairs in the cluster. Thus from (??) and (13) we can determine all the correlation functions which are necessary for the calculation of the concurrence.

The partition function is found from the following formula

$$
Z=\sum_{S=0, \frac{1}{2}}^{\frac{N}{2}} \sum_{m=-S}^{S} e^{-\beta \frac{J}{N-1}\left(S(S+1)+\Delta m^{2}\right)+B m} g(N, S)
$$

where we have ignored a multiplicative constant and the lower limit of the sum begins from $S=0\left(S=\frac{1}{2}\right)$ for even (odd) $N$ and

$$
g(N, S)=\left(\begin{array}{c}
N \\
\frac{N}{2}-S
\end{array}\right) \frac{2 S+1}{\frac{N}{2}+S+1}
$$


is the number of times a spin $S$ representation appears in the decomposition of the tensor product of $N$ copies of spin $\frac{1}{2}$ representations. Note that we have to make a distinction between odd and even number of sites $N$, since for even $N$ (odd $N$ ) only integer (half-integer) spins appear in the decomposition. We have now set up the required equations and are ready to analyze various situations. First we consider the isotropic model in zero magnetic field.

\section{The xxx model in zero magnetic field}

In this case we have $B=\Delta=0$. For a finite cluster we have no spontaneous magnetization and hence $\mu=0$. Moreover by rotational symmetry we have $G_{x x}=G_{y y}=G_{z z}=\frac{\varepsilon}{3 J}$. Therefore we find from (9) that

$$
C=2 \max \left[0,\left|\frac{2}{3 J} \varepsilon\right|-\left|\frac{1}{4}+\frac{\varepsilon}{3 J}\right|\right]=2 \max \left[0, \frac{2}{3}|x|-\left|\frac{1}{4}+\frac{x}{3}\right|\right],
$$

where we have abbreviated $\frac{\varepsilon}{J}$ to $x$. We now note that any state with total spin $S$ has an energy given by

$$
E_{S}=\frac{J}{N-1}\left(S(S+1)-\frac{3}{4} N\right)
$$

from which we can derive a bound for the variable $x=\frac{\varepsilon}{J}$. This bound is obtained by considering the maximum and the minimum values of $S$, respectively given by $\frac{N}{2}$ and $0\left(\frac{1}{2}\right)$ for even (odd) $N$.

$$
\begin{aligned}
-\frac{3}{4(N-1)} \leq x \leq \frac{1}{4} & \text { for } & N & =\text { even } \\
-\frac{3}{4 N} \leq x \leq \frac{1}{4} & \text { for } & N & =\text { odd }
\end{aligned}
$$

On the other hand it is readily seen that the function $f(x):=\frac{2}{3}|x|-\left|\frac{1}{4}+\frac{x}{3}\right|$ satisfies the following bound

$$
0<f(x) \quad \text { only if } \quad x<\frac{-1}{4} .
$$

Combination of (18) and (19) shows that there is no entanglement for any isotropic cluster for $N \geq 3$. We can also exclude the ferromagnetic $N=2$ cluster by noting that for such a cluster $\varepsilon \leq 0$ (sine at infinitely high temperatures $\varepsilon \propto \operatorname{tr}(H)=0$, consequently at lower temperatures $\varepsilon \leq 0)$ and $J \leq 0$ making the fraction $x$ a positive number. Thus we arrive at the result that

The only isotropic mean field cluster which can be entangled at non-zero temperature is the $N=2$ anti-ferromagnetic cluster.

It had already been shown [6] that in the class of anti-ferromagnetic rings, the $N=3$ case does not show entanglement, here we see that this is a special case of a more general result which holds for all mean field clusters, including $N=3$ clusters as a special case. 


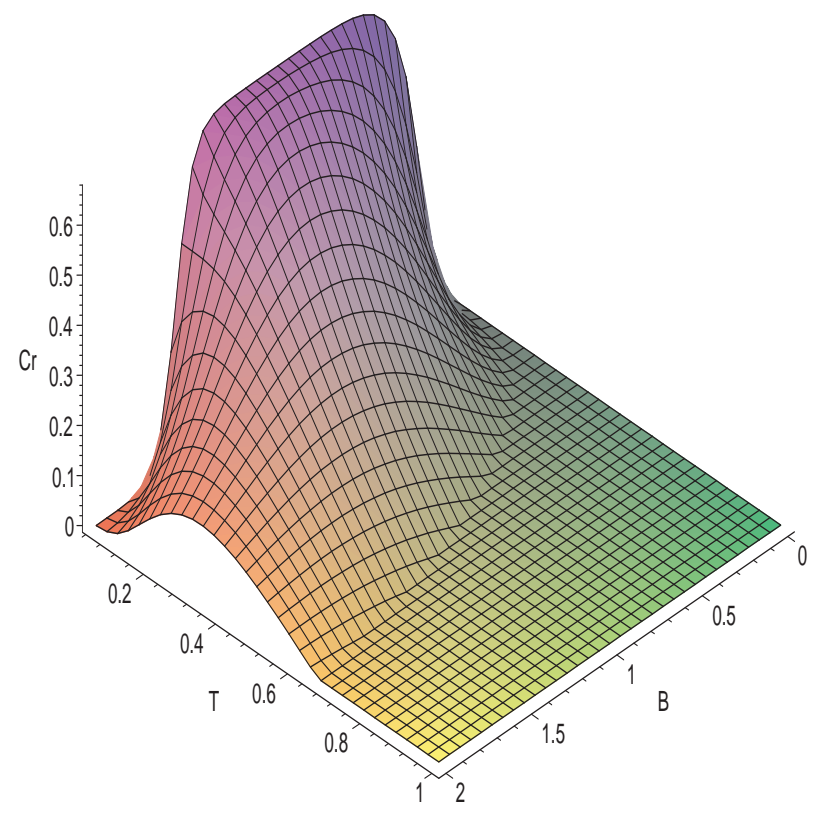

Figure 1: Color online. The re-scaled concurrence for an $N=3 \mathrm{xxx}$ cluster, a triangle of anti-ferromagnetic spins, as a function of temperature and magnetic field. In this and all the other figures, the units are so chosen that $B$ and $T$ become dimensionless. The concurrence is a dimensionless quantity.

\section{The $\mathrm{xxx}$ model in magnetic field}

In the presence of magnetic field, the model will no longer have the $s u(2)$ symmetry and the relation $G_{x x}=G_{y y}=G_{z z}=\frac{ \pm \varepsilon}{3}$ no longer holds. However one can use (14) and calculate the partition function which now takes the form

$$
Z=\sum_{S=0, \frac{1}{2}}^{\frac{N}{2}} e^{-\beta \frac{J}{N-1} S(S+1)} \frac{\sinh \beta B\left(S+\frac{1}{2}\right)}{\sinh \frac{\beta B}{2}} g(N, S) .
$$

All the required correlation functions can be obtained exactly, but can not be written in terms of short expressions. The concurrence is obtained numerically. The result is that no thermal entanglement develops in the ferromagnetic clusters but for anti-ferromagnetic clusters, there is entanglement which generally but not always diminishes by increasing temperature. Figures (11) and (2) show the thermal entanglement of $N=3$ and $N=4$ clusters as functions of temperature and magnetic field. In both of them we see that there are regions in the $B-T$ plane where an increase of temperature first increases the entanglement and then tends to decease the entanglement. The maximum entanglement exists at zero temperature and inside a certain interval of magnetic field values.

In order to depict the typical information contained in these two figures for different cluster sizes, we refer the reader to figures (3) and (44). Figure (3) shows the entanglement at very low 


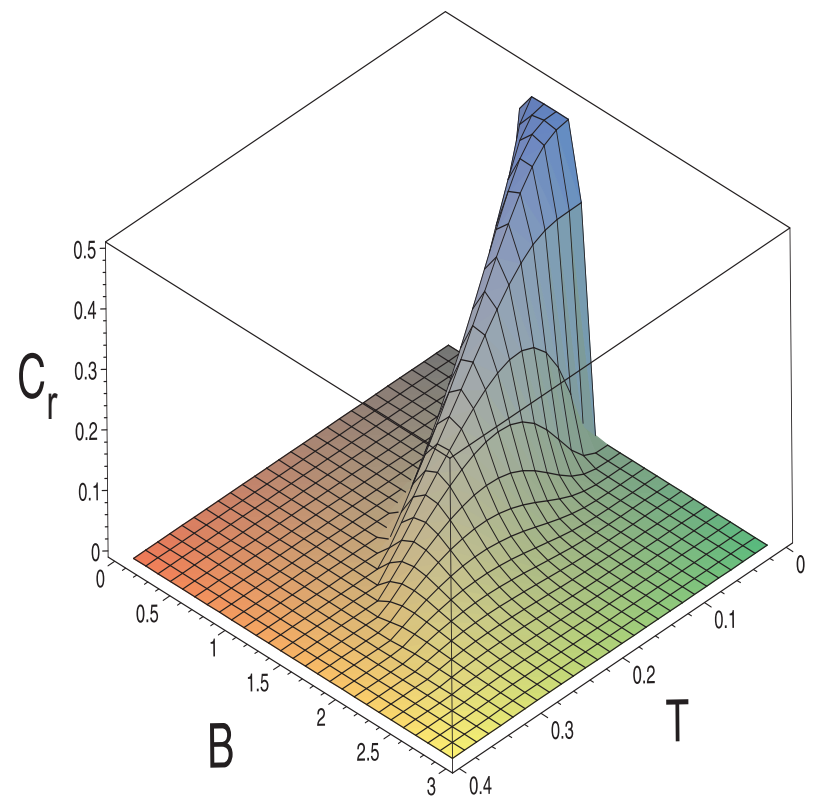

Figure 2: Color online. The re-scaled concurrence for an $N=4 \mathrm{xxx}$ cluster, a tetrahedron of anti-ferromagnetic spins, as a function of temperature and magnetic field.

temperatures $(\mathrm{T}=0.01)$ for $x x x$ clusters as a function of magnetic field $B$, for cluster sizes. It is seen that by increasing the size of clusters, the interval for which there is entanglement shrinks to a vanishingly small interval centered around $B=1$. Figure (44) shows the region in the $B-T$ plane in which the cluster is entangled. The region becomes smaller and smaller as the size of the cluster increases. The right-tilted shape of these regions indicates that in certain intervals of magnetic fields one can generate entanglement simply by increasing the temperature. The physical reason is that when the magnetic field is high, the ground state is a state with all the spins aligned in the direction of magnetic field and hence there is no entanglement at zero temperature. Slightly increasing the temperature mixes the entangled excited states with the ground state and generates entanglement.

Finally figure (5) shows the maximum re-scaled concurrence for clusters of different sizes. By re-scaled concurrence we mean the concurrence between two sites multiplied by $N-1$ which is the number of neighbors of a given site. It is seen that there is almost no entanglement for clusters of size larger than 23.

This concludes our investigation of the $x x x$ clusters in magnetic field. We now turn to another type of anisotropy, namely the $x x z$ clusters in which the anisotropy is not brought about by an external magnetic field. 


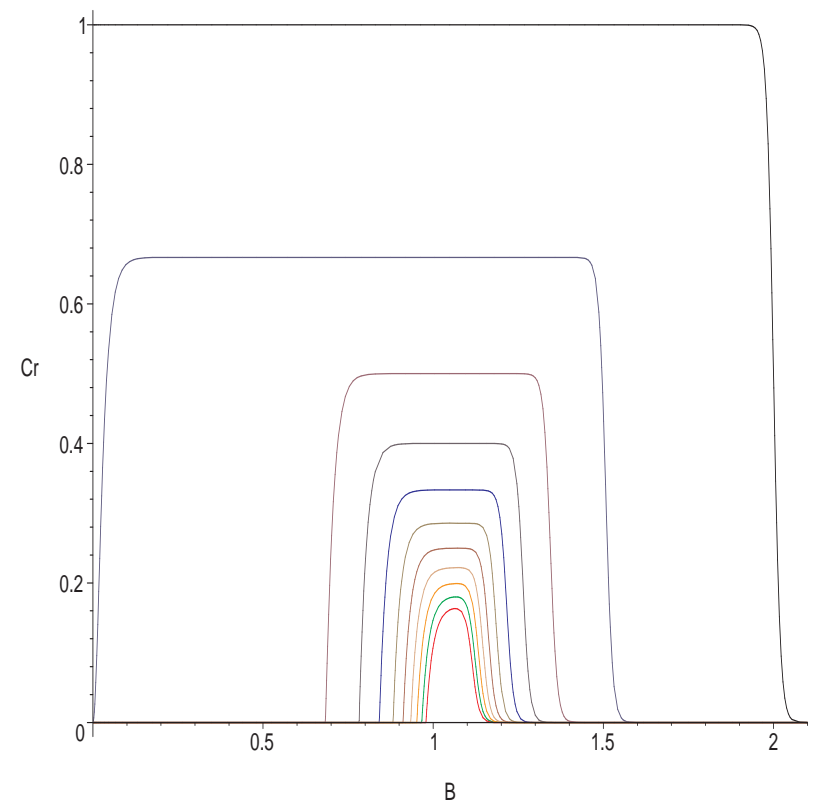

Figure 3: Color online. The re-scaled concurrence for different mean clusters at very low temperatures $(\mathrm{T}=0.01)$ as a function of magnetic field. The curves correspond (from top to bottom) to $(N=2)$ to $(N=12)$. The units are so chosen that $B$ and $T$ become dimensionless. The concurrence is a dimensionless quantity.

\section{The $x x z$ model in zero magnetic field}

For these clusters we find a surprising results: Except for $N=2$, the anti-ferromagnetic clusters show no entanglement under any condition, regardless of the value $\Delta$ and $T$. However ferromagnetic clusters show entanglement for all negative values of the anisotropy parameter $\Delta$. We do not report the result for the $N=2$ ferromagnetic cluster, since this has been reported elsewhere 13 and focus instead on clusters of arbitrary sizes.

The entanglement of a ferromagnetic cluster of size $N=20$ is shown in figure ([6). This figure is typical, that is all the ferromagnetic clusters have a similar behavior.

In order to depict the relevant information for clusters of different sizes we refer the reader to figures (9) through (11). Figure (91) and (10) show the entanglement at very low temperature, i.e. $T=0.01$, for even and odd size clusters as a function of $\Delta$. Note the difference in trends, that is, for even size clusters as $N$ increases the re-scaled concurrence decreases while for odd size clusters, as $N$ increases the re-scaled concurrence increases. The two figures suggest that in the limit $N \longrightarrow \infty$, the re-scaled concurrence approaches a curve which we redraw in figure (111). The insets in these figures i.e. figures (9) and (10) show the regions in the $\Delta-T$ plane below which thermal entanglement exists for different clusters. For both even and odd size clusters, as the size of the cluster increases, the regions decrease in size but do not shrink completely and approach a limiting region. 


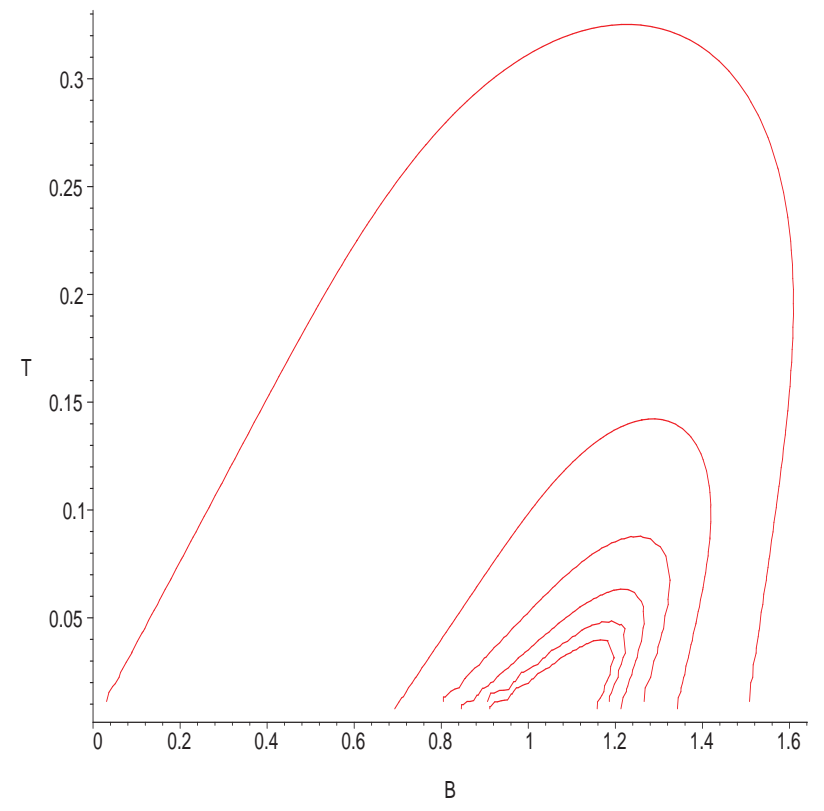

Figure 4: The regions of entanglement in the B-T plane for mean field clusters of size $\mathrm{N}=3$ to size $\mathrm{N}=8$.

We have to understand two important characteristics of the entanglement of these clusters. First why in the limit $\Delta \longrightarrow \infty$, where the interaction approaches an Ising-like interaction we still have entanglement at very low temperatures and second why in the anti-ferromagnetic case there is no entanglement at all for any value of $\Delta$. To this order we present a simple examples, namely the $=3$ cluster.

\subsection{The $\mathrm{N}=3$ cluster}

For such a cluster, the hamiltonian is

$$
H=J\left(s_{1} \cdot s_{2}+s_{2} \cdot s_{3}+s_{1} \cdot s_{3}\right)+J \Delta\left(s_{1 z} s_{2 z}+s_{1 z} s_{3 z}+s_{2 z} s_{3 z}\right) \equiv J S \cdot S+J \Delta S_{z}^{2},
$$

where in the second form, we have ignored an additive constant. Any state of the form $|s, m\rangle$ where $s$ and $m$ are respectively the total and the $z$ component of the total spin is an eigenstate of energy with energy given by $E_{s, m}=J\left(s(s+1)+\Delta m^{2}\right)$. The eigenstates are as follows:

$$
\begin{aligned}
&\left|\frac{3}{2}, \frac{3}{2}\right\rangle=|+,+,+\rangle, \\
&\left|\frac{3}{2}, \frac{1}{2}\right\rangle=\frac{1}{\sqrt{3}}(|+,+,-\rangle+|+,-,+\rangle+|-,+,+\rangle), \\
&\left|\frac{3}{2}, \frac{-1}{2}\right\rangle=\frac{1}{\sqrt{3}}(|-,-,+\rangle+|-,+,-\rangle+|+,-,-\rangle),
\end{aligned}
$$




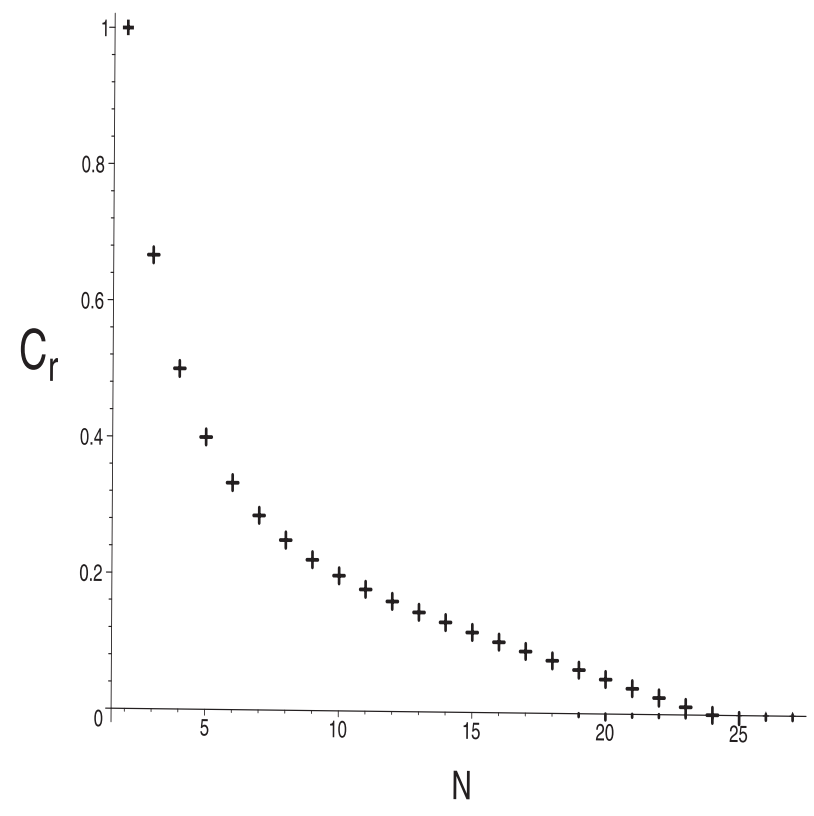

Figure 5: Maximum re-scaled concurrence for isotropic mean field clusters in magnetic field as a function of their size $N$. For $N>23$, the concurrence vanishes.

$$
\begin{aligned}
\left|\frac{3}{2}, \frac{-3}{2}\right\rangle=|-,-,-\rangle, \\
\left|\frac{1}{2}, \frac{1}{2}\right\rangle=\frac{1}{\sqrt{2}}(|+,-,+\rangle+|-,+,+\rangle), \\
\left|\frac{1}{2}, \frac{-1}{2}\right\rangle=\frac{1}{\sqrt{2}}(|+,-,-\rangle+|-,+,-\rangle),
\end{aligned}
$$

and

$$
\begin{aligned}
& \left|\frac{1^{\prime}}{2}, \frac{1}{2}\right\rangle=\frac{1}{\sqrt{6}}(|-,+,+\rangle+|+,-,+\rangle-2|+,+,-\rangle), \\
& \left|\frac{1^{\prime}}{2}, \frac{-1}{2}\right\rangle=\frac{1}{\sqrt{6}}(|+,-,-\rangle+|-,+,-\rangle+|-,-,+\rangle) .
\end{aligned}
$$

The energies of the states are one of the values $\frac{J}{4}(15+9 \Delta), \frac{J}{4}(15+\Delta)$ and $\frac{J}{4}(3+\Delta)$, depending on the values of the quantum numbers $s$ and $m$.

Figures (8) and (77) show the spectrum in the ferromagnetic and anti-ferromagnetic cases separately. In each case the nature of spectrum depends on the value of $\Delta$. Let us consider the ferromagnetic and anti-ferromagnetic cases separately. 


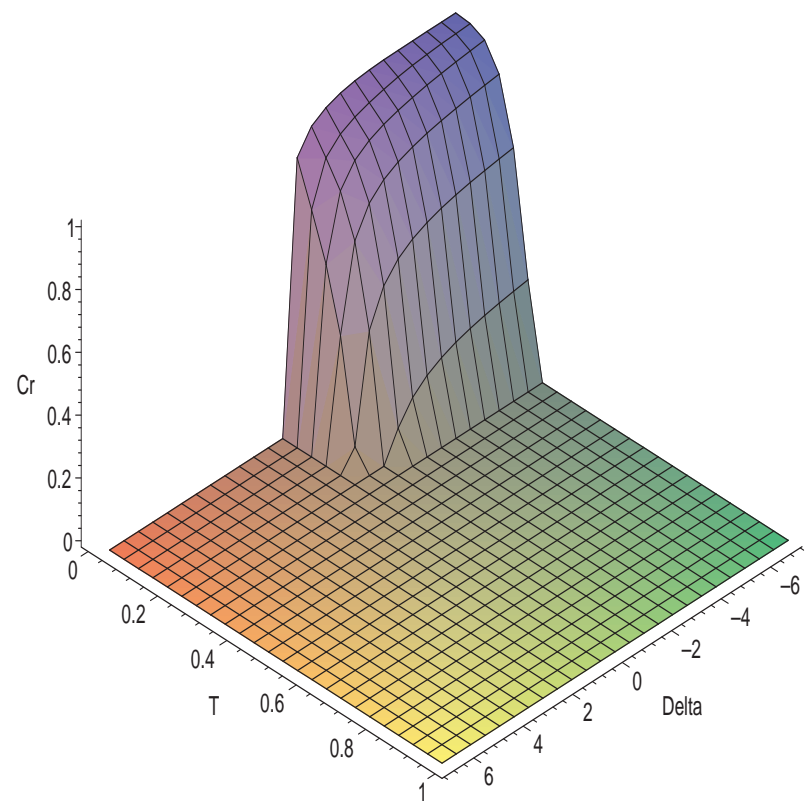

Figure 6: Color online. Re-scaled concurrence for an $N=20 x x z$ mean field cluster as a function of temperature and anisotropy.

\section{Anti-ferromagnetic interaction: $J=1$}

Here we have the ground state energy $E_{g s}$ as

$$
E_{g s}=\left\{\begin{array}{lll}
\frac{1}{4}(15+9 \Delta) & \text { for } & \Delta<\frac{-3}{2}, \\
\frac{1}{4}(3+\Delta) & \text { for } & \frac{-3}{2} \leq \Delta
\end{array}\right.
$$

Consequently for $\Delta<\frac{-3}{2}$, the thermal state at zero temperature is equal to

$$
\rho(T=0)=\frac{1}{2}(|+,+,+\rangle\langle+,+,+|+|-,-,-\rangle\langle-,-,-|)
$$

which is clearly a separable state. For $\frac{-3}{2} \leq \Delta$, the thermal state is an equal mixture of the four states with $s=\frac{1}{2}$. A simple calculation gives in this case, the explicit form of the two particle density matrix as

$$
\rho(T=0)=\frac{1}{6}\left(\begin{array}{cccc}
1 & & & \\
& 2 & -1 & \\
& -1 & 2 & \\
& & & 1
\end{array}\right)
$$

with eigenvalues $\frac{1}{6}, \frac{1}{6}, \frac{1}{6}$ and $\frac{1}{2}$. Since in this case we have $\sqrt{\rho \tilde{\rho}}:=\sqrt{\rho\left(\sigma_{y} \otimes \sigma_{y}\right) \rho^{*}\left(\sigma_{y} \otimes \sigma_{y}\right)}=$ $\rho$, we find the concurrence of this state to be 


$$
\begin{aligned}
& \left|\frac{3}{2}, \pm \frac{1}{2}\right\rangle \longrightarrow \frac{1}{4}(15+\Delta) \quad\left|\frac{3}{2}, \pm \frac{1}{2}\right\rangle \longrightarrow \frac{1}{4}(15+\Delta) \\
& \left|\frac{1}{2}, \pm \frac{1}{2}\right\rangle \longrightarrow \frac{1}{4}(3+\Delta) \quad\left|\frac{3}{2}, \pm \frac{3}{2}\right\rangle \longrightarrow \frac{1}{4}(15+9 \Delta) \\
& \left|\frac{3}{2}, \pm \frac{3}{2}\right\rangle \longrightarrow \frac{1}{4}(15+9 \Delta) \quad\left|\frac{1}{2}, \pm \frac{1}{2}\right\rangle \longrightarrow \frac{1}{4}(3+\Delta) \\
& \Delta<-\frac{3}{2} \quad \mathrm{~J}=1 \quad \Delta \geq-\frac{3}{2}
\end{aligned}
$$

Figure 7: The spectrum of the $N=3 x x z$ anti-ferromagnetic cluster. Note that $\left|\frac{1}{2}, \frac{1}{2}\right\rangle$ stands for two doublets.

$$
\begin{aligned}
& \left|\frac{1}{2}, \pm \frac{1}{2}\right\rangle \longrightarrow \frac{1}{4}(3+\Delta) \quad\left|\frac{1}{2}, \pm \frac{1}{2}\right\rangle \longrightarrow \frac{1}{4}(3+\Delta) \\
& \left|\frac{3}{2}, \pm \frac{3}{2}\right\rangle \longrightarrow \frac{1}{4}(15+9 \Delta) \quad\left|\frac{3}{2}, \pm \frac{1}{2}\right\rangle \longrightarrow \frac{1}{4}(15+\Delta) \\
& \left|\frac{3}{2}, \pm \frac{1}{2}\right\rangle \longrightarrow \frac{1}{4}(15+\Delta) \quad\left|\frac{3}{2}, \pm \frac{3}{2}\right\rangle \longrightarrow \frac{1}{4}(15+9 \Delta) \\
& \Delta<0 \quad J=-1 \quad \Delta \geq 0
\end{aligned}
$$

Figure 8: The spectrum of the $N=3 x x z$ ferromagnetic cluster. Note that $\left|\frac{1}{2}, \frac{1}{2}\right\rangle$ stands for two doublets.

$$
C=\max \left(0, \frac{1}{2}-3 \frac{1}{6}\right)=0 .
$$

Therefore we have shown that this anti-ferromagnetic cluster is not entangled for any value of the anisotropy.

Here we have used the original form of the concurrence [1] valid for any density matrix of two qubits which states that

$$
C=\max \left(0, \lambda_{1}-\lambda_{2}-\lambda_{3}-\lambda_{4}\right),
$$

where $\lambda_{i}$ 's are the positive square roots of the eigenvalues of $\rho \tilde{\rho}$.

The ferromagnetic interaction: $J=-1$ 


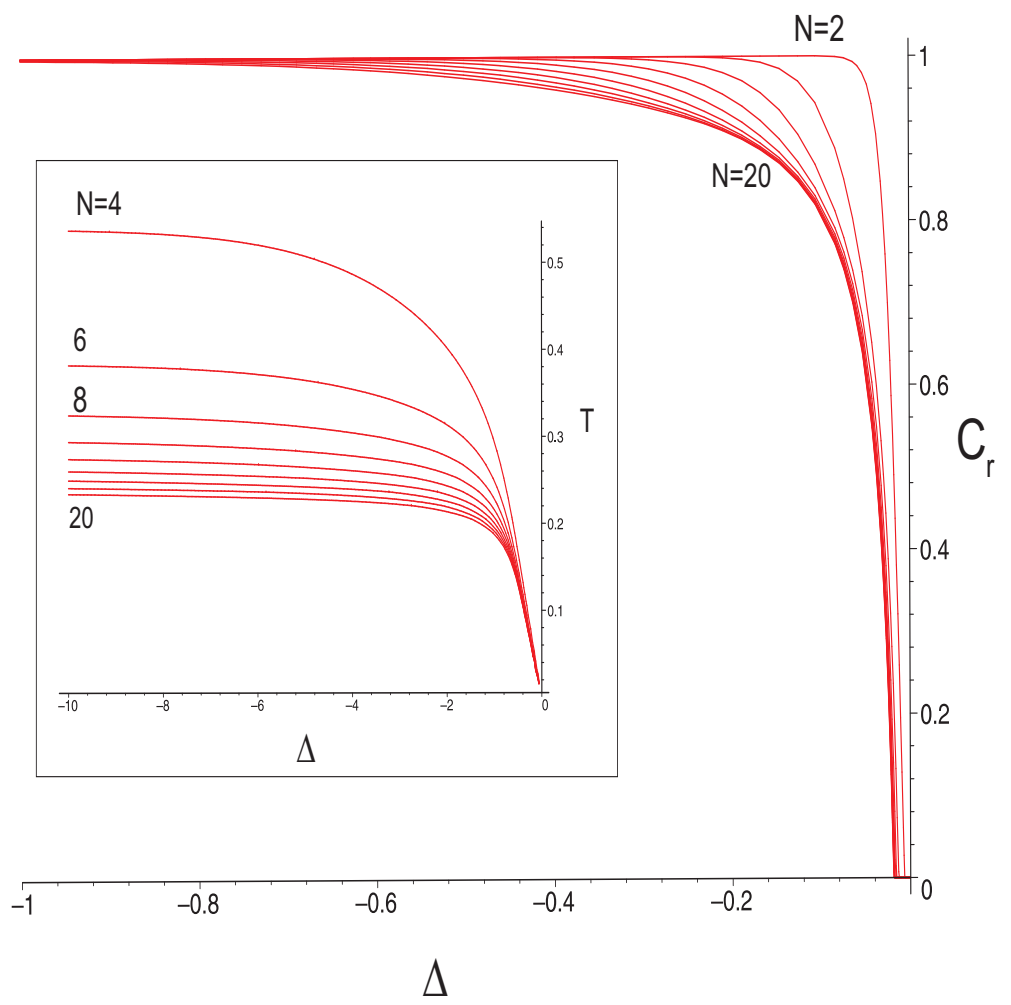

Figure 9: Re-scaled concurrence for anisotropic mean field clusters at nearly zero temperature ( $\mathrm{T}=0.01$ ), for clusters of even size, from $\mathrm{N}=2$ to $\mathrm{N}=20$. The inset shows the regions in the $\Delta-T$ plane below which there is entanglement. The units are so chosen that $T$ is dimensionless. The parameter $\Delta$ and the concurrence $C_{r}$ are dimensionless quantities.

In this case the ground state energy $E_{g s}$ is

$$
E_{g s}=\left\{\begin{array}{lll}
\frac{-1}{4}(15+\Delta) & \text { for } & \Delta<0, \\
\frac{-1}{4}(15+9 \Delta) & \text { for } & 0 \leq \Delta
\end{array}\right.
$$

Consequently for $\Delta>0$, the thermal state at zero temperature $\rho(T=0)$ is equal to

$$
\rho(T=0)=\frac{1}{2}(|+,+,+\rangle\langle+,+,+|+|-,-,-\rangle\langle-,-,-|)
$$

which is clearly a separable state. On the other hand For $\Delta \leq 0$, the thermal state is an equal mixture of the two states with $\left|\frac{3}{2}, \frac{1}{2}\right\rangle$ and $\left|\frac{3}{2}, \frac{-1}{2}\right\rangle$. A simple calculation gives in this case, the explicit form of the two particle density matrix as

$$
\rho(T=0)=\frac{1}{6}\left(\begin{array}{llll}
1 & & & \\
& 2 & 2 & \\
& 2 & 2 & \\
& & & 1
\end{array}\right),
$$




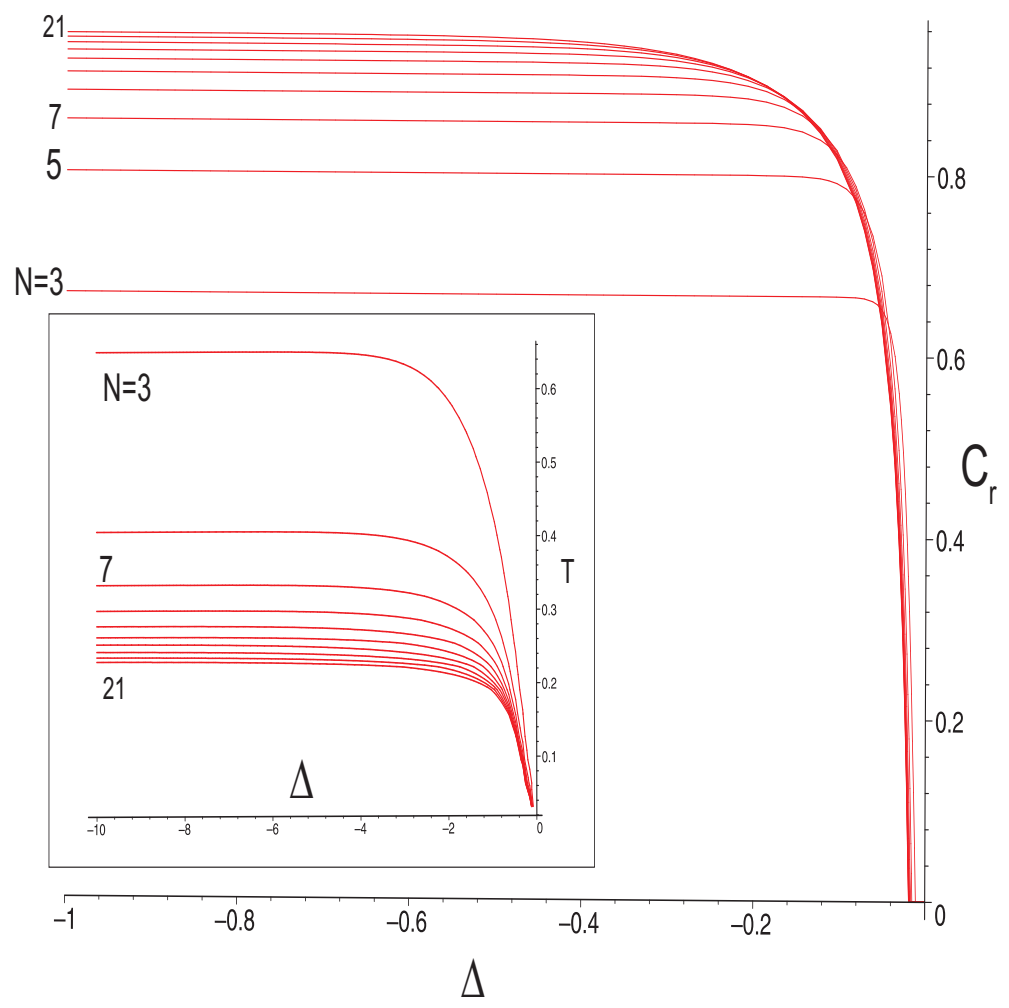

Figure 10: Re-scaled concurrence for anisotropic mean field clusters at nearly zero temperature ( $\mathrm{T}=0.01$ ), for clusters of odd size, from $\mathrm{N}=3$ to $\mathrm{N}=21$. The inset shows the regions in the $\Delta-T$ plane below which there is entanglement.)

with eigenvalues $\frac{1}{6}, \frac{1}{6}, \frac{4}{6}$ and 0 . This gives the concurrence to be

$$
C=\max \left(0, \frac{4}{6}-2 \frac{1}{6}\right)=\frac{1}{3},
$$

Therefore this cluster is entangled only for negative values of $\Delta$ and for ferromagnetic interaction.

Let us see why at zero or very low temperature a ferromagnetic cluster shows entanglement in the limit of very large and negative $\Delta$, despite the Ising like appearance of the interaction. The reason is that in this case, the Hamiltonian approaches $H=-\left(S^{2}+\Delta S_{z}^{2}\right) \approx-\Delta S_{z}^{2}$. Since $\Delta<0$, the lowest energy states are those with $S_{z}= \pm \frac{1}{2}$, and these states, as shown in figure (8) are the states $\left|\frac{3}{2}, \pm \frac{1}{2}\right\rangle$ which are highly entangled. One may now ask why the same phenomena does not happen in the anti-ferromagnetic case for very large positive $\Delta$, for which the Hamiltonian takes a similar form? The reason is that as shown in figure (7) the ground state is now a mixture of four $S_{z}= \pm \frac{1}{2}$ states, and this mixture does not have any entanglement, although the states themselves may be entangled. 


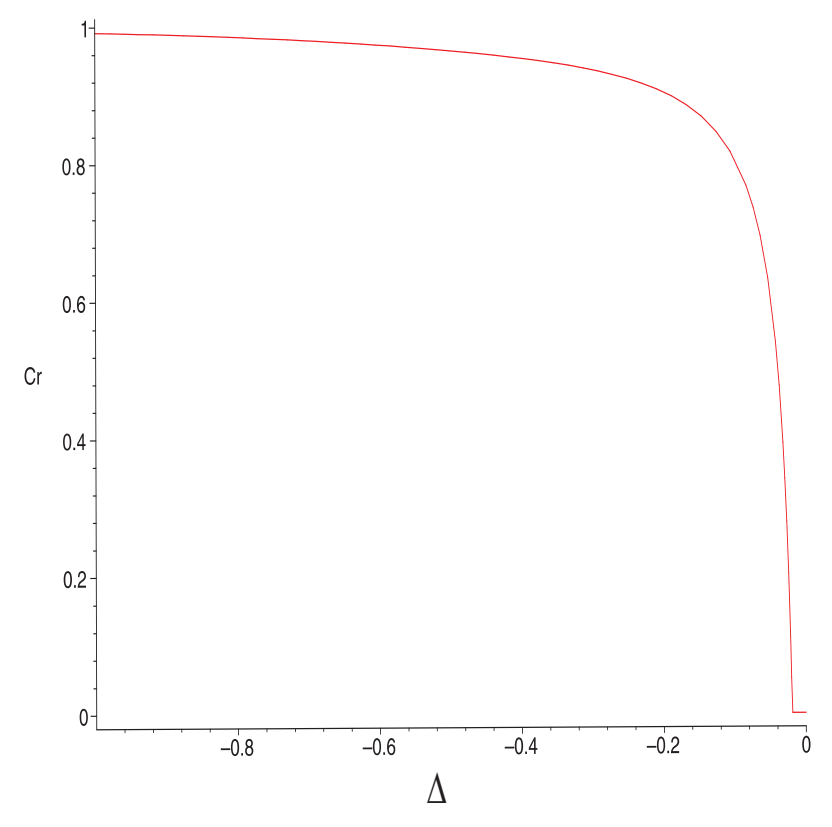

Figure 11: The re-scaled concurrence for an $x x z$ mean field cluster in the limit of large $N$ as a function of $\Delta$, at very low temperature $T=0.01$

\section{Discussion}

We have done a rather detailed study of the pairwise entanglement properties of a mean field cluster of spin one-half particles interacting via the Heisenberg $x x z$ interaction. We have considered the ferromagnetic and anti-ferromagnetic interactions and the isotropic $(x x x)$ and anisotropic $(x x z)$ cases with and without magnetic field. In each case we have determined the dependence of entanglement on various parameters like the external magnetic field, the anisotropy parameter, the cluster size as well as temperature.

It has already been shown that, although the two particle concurrence of the ground state vanishes in the thermodynamic limit, the re-scaled concurrence does not vanish and shows quite interesting and nontrivial behavior [27, 28, 29, 30]. Some of our results (figures (9) and (10)) shows that as the size of clusters approach infinity, the re-scaled concurrence at non-zero temperature approaches a specific function of the control parameters. In a future work we will focus on the thermodynamic limit of these clusters to investigate these functions where we should also take into account the possibility of spontaneous magnetization and symmetry breaking.

\section{Acknowledgement}

We would like to thank the members of the Quantum information group of Sharif University for very valuable comments. 


\section{References}

[1] S. Hill and W. K. Wootters, Phys. Rev. Lett. 78, 5022 (1997); W. K. Wootters, Phys. Rev. Letts. 80, 2245 (1998).

[2] M. A. Nielson, PhD thesis, University of New Mexico(1998), quant-ph/0011036.

[3] M. C. Arnesen, S. Bose, and V. Vedral, Phys. Rev. Lett. 87, 277901 (2001).

[4] D. Gunlycke et al, Phys. Rev. A 64, 042302 (2001).

[5] M. K. O'Connor and W. K. Wootters, Phys. Rev. A 63, 052302 (2001).

[6] X. Wang, and P. Zanardi, Phys. Lett. A 301 (1-2), 1 (2002).

[7] I. Bose and A. Tribedi, Phys. Rev. A 72, 022314 (2005).

[8] M. Asoudeh, and V. Karimipour, Phys. Rev. A 70, 052307 (2004).

[9] M. Asoudeh, and V. Karimipour, Phys. Rev. A, 71, 022308 (2005).

[10] G. L. Kamta and A. F. Starace, Phys. Rev. Lett. 88, 107901 (2002).

[11] N. Canosa and R. Rossignoli, Phys. Rev. A 69, 052306 (2004).

[12] Y. Sun, Y. Chen and H. Chen, Phys. Rev. A 68, 044301 (2003).

[13] G. Rigolin, Int. J. Quant. Inf. 2, 393 (2004).

[14] X. Wang, Phys. Rev. A 66, 044305 (2002).

[15] X. Wang, Phys. Rev. A 66, 034302 (2002).

[16] X. Wang, H. Fu, and Allan I. Solomon, J. Phys. A: Math. Gen. 34(50), 11307-11320(2001).

[17] B. E. Kane, Nature, 393133 (1998).

[18] D. Loss and D. P. DiVincenzo, Phys. Rev. A 57, 120(1998); J. Levy, Phys. Rev. A 64, 052306 (2001).

[19] DiVincenzo et al, NATURE, Vol. 408, p. 339-342 (2000).

[20] G. Burkard and G. Loss, Phys. Rev. Lett. 88, 047903 (2002).

[21] A. Imamoglu et al, Phys. Rev. Lett. 83, 4204 (1999).

[22] K. V. Kavokin, Phys. Rev. B 64, 075305 (2001).

[23] L. A. Wu and D. A. Lidar, Phys. Rev. A 66, 062314 (2002). 
[24] J. Vidal, R. Mosseri, and J. Dukelsky, Phys. Rev. A 69, 054101 (2004).

[25] J. Vidal, G. Palacios, and R. Mosseri, Phys. Rev. A 69, 022107 (2004).

[26] H. J. Lipkin, N. Meshkov and A. J. Click, Nucl. Phys. 62199 (1965); N. Meshkov and A. J. Click, and H. J. Lipkin, Nucl. Phys. 62188 (1965); A. J. Click, H. J. Lipkin and N. Meshkov, Nucl. Phys. 62211 (1965).

[27] J. Vidal, Concurrence in collective models, quant-ph/0603108.

[28] J. I. Latorre, R. Orus, E. Rico and J. Vidal, Phys. Rev. A 71, 064101 (2005).

[29] S. Dusuel and J. Vidal, Phys. Rev. Lett. 93, 237204 (2004).

[30] S. Dusuel and J. Vidal, Phys. Rev. B 71, 224420 (2005). 